\title{
Two-wave pattern shift aberration monitor for centrally obscured optical systems
}

\author{
Jason P. Cain, ${ }^{a *}$ Gregory McIntyre, ${ }^{a}$ Patrick Naulleau, ${ }^{b}$ Adam Pawloski, ${ }^{c}$ Bruno La Fontaine ${ }^{c}$ \\ Obert Wood, ${ }^{c}$ Costas J. Spanos, ${ }^{a}$ Andrew R. Neureuther ${ }^{a}$ \\ ${ }^{a}$ Department of Electrical Engineering and Computer Sciences, \\ University of California, Berkeley, CA 94720 \\ ${ }^{b}$ Center for X-Ray Optics, Lawrence Berkeley National Laboratory, Berkeley, CA 94720 \\ ${ }^{c}$ Advanced Micro Devices, Sunnyvale, CA 94088
}

\begin{abstract}
An aberration monitoring technique based on lateral shifts of two-wave interference patterns in centrally obscured optical systems is presented, and simulations are used to evaluate the performance of such a technique. The technique is being explored as a convenient means for monitoring the aberration level in the 0.3-NA Micro Exposure Tool (MET) optic over time. A binary mask was designed for observing phase differences across the MET optic on cut-lines at 0,45 , 90 and 135 degrees across the pupil. The mask consists of 5 line-and space patterns in a dark field that measure the sideto-side phase difference across the pupil at 7 equally spaced radial points extending from $35 \%$ to $95 \%$ of the pupil radius. For near on-axis illumination the blockage of the zero-order creates a two-wave, interferometric pattern at the wafer with half of the period expected under normal imaging conditions. The optical path difference between the two orders produces an image shift of one full period of the (frequency doubled) interference pattern per 360 degrees of sideto-side path difference. Shifts on the order of 5 to $20 \mathrm{~nm}$ are expected and are measured using a reference target of an array of 5 medium sized dots. Aerial image simulation is being utilized to predict the expected performance and to improve the initial design. The aberrations measured by interferometry are being used for this purpose. Also the quality of images at low partial coherence with the wavefront convergence present in the MET illumination is being studied. In addition to theory and simulation results, practical considerations in implementing this technique on actual lithography tools based upon MET-type optics are addressed, including pattern design, illumination characteristics, and data analysis.
\end{abstract}

Keywords: Extreme ultraviolet (EUV) lithography, aberrations, central obscuration, micro-exposure tool (MET) optic

\section{INTRODUCTION}

Conventional optical lithography is widely believed to be a non-manufacturable solution at the $32 \mathrm{~nm}$ technology node and beyond, and several different potential next-generation lithography (NGL) technologies have been the subject of active research for many years. One such technology is lithography at extreme ultraviolet (EUV) wavelengths around $13.5 \mathrm{~nm}$. Transitioning from deep UV to EUV wavelengths requires much more than simply a shorter-wavelength source, as the absorptive nature of virtually all materials in this region of the electromagnetic spectrum leads to a wide range of other difficulties. In spite of these difficulties, a great deal of progress in this field has been made in the past few years.

In order to investigate issues related to EUV lithography development, a static micro-field exposure tool based on the Micro-Exposure Tool (MET) optic and operating at a wavelength of $13.5 \mathrm{~nm}$ has been installed at the Advanced Light Source, a synchrotron facility at the Lawrence Berkeley National Laboratory. ${ }^{1-3}$ The MET optic is composed of two multilayer-coated reflective elements and has a numerical aperture (NA) of 0.3 , comparable to the value expected for first-generation EUV production tools. The optic has an annular pupil with a central obscuration of NA 0.1. The field size is $600 \mu \mathrm{m} \times 200 \mu \mathrm{m}$ at the wafer, and the tool uses a scanning illuminator to provide programmable coherence control. ${ }^{4}$ This is highly advantageous as it allows for a many different illumination types and a partial coherence $(\sigma)$ range of roughly 0.05 to 1 .

\footnotetext{
${ }^{*}$ Further author information: (Send correspondence to J.P.C., now with Advanced Micro Devices) J.P.C.: E-mail: jason.cain@amd.com, Telephone: 1 (408) 749-2609
} 
One area of concern in EUV lithography is the quality of the projection optics with regard to aberrations and flare. In this work, a method for monitoring drift in the level of aberrations present in an optic with an annular pupil (such as the MET optic) is presented. The method is described in detail in Section 2. A mask to test this technique has been designed and fabricated, and this is presented in Section 3. Practical considerations in the actual implementation of the test method are discussed in Section 4. Finally, conclusions are given in Section 5.

\section{DESCRIPTION OF MEASUREMENT TECHNIQUE}

This method uses line/space patterns and highly coherent illumination $(\sigma \cong 0.05)$ to create diffracted orders that "sample" the wavefront of the projection optics. The zero order is entirely blocked by the central obscuration, and the pitch is chosen so that the first orders pass through the lens at a specified radius within the pupil. These two waves will then interfere at the wafer plane to create the aerial image, which is then transferred into photoresist. If aberrations are present in the lens, then an optical path difference (OPD) will exist between the two waves. As shown in Figure 1, this may be thought of as causing a "tilt" in the wavefront. This tilt will result in a lateral shift of the aerial image at the wafer. By measuring the lateral shift as a function of pitch (and therefore a function of pupil position), the "signature" of a particular set of aberrations may be recorded. Changes in this signature over time indicate a drift in the aberrations present in the system.

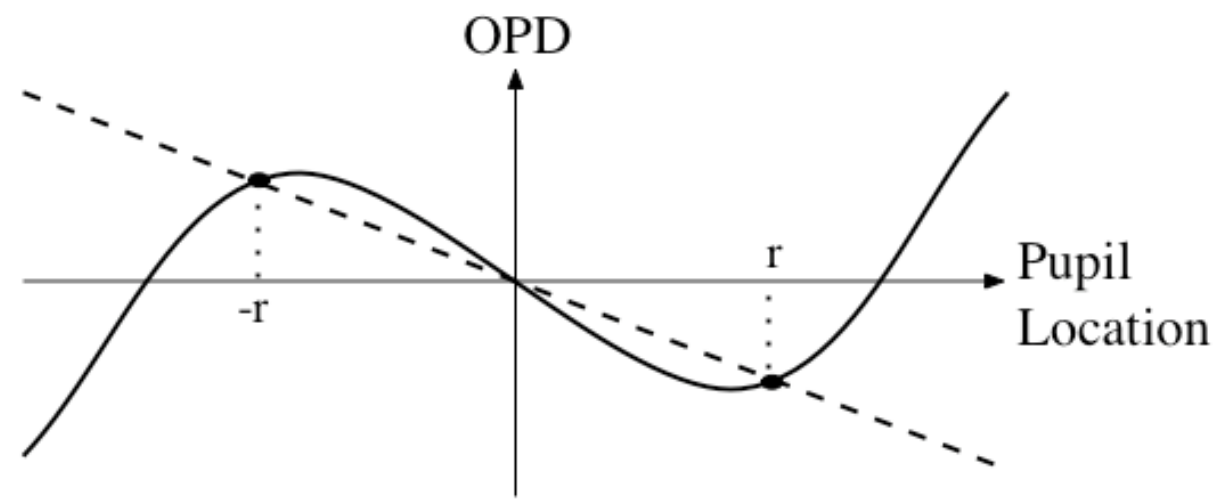

Figure 1. The interference of two waves with a non-zero optical path difference (OPD) may be interpreted as a tilted wavefront, resulting in a lateral shift of the aerial image at the wafer.

The blocking of the zero order in this method has one important consequence: the aerial image becomes frequencydoubled relative to the mask pattern. The process by which this occurs is illustrated in Figure 2 . The aerial image electric field and intensity for the case with no central obscuration are shown In Figure 2a.) and b.), respectively. Here the aerial image intensity, $I$, is given by $I=E^{*} E$, where $E$ is the aerial image electric field. The aerial image electric field and intensity for the case with the central obscuration are shown in Figure 2c.) and d.), respectively.

The amount of lateral shift expected in the aerial image for a given OPD may be deduced by noting that the difference in phase between the two waves interfering at the wafer goes from $0^{\circ}$ to $180^{\circ}$ (one half-wave) over a lateral distance of $P_{\text {wafer }} / 2$, where $P_{\text {wafer }}$ is the pitch of the aerial image at the wafer. Due to the frequency doubling which occurs when the zero order is blocked, $P_{\text {wafer }}=P_{\text {mask }} / 2$. Therefore, the amount of lateral shift $\Delta x$ which will occur for an OPD $\Delta \phi$ in waves as a function of normalized pupil radius $r_{p u p i l}$ is given by

$$
\Delta x\left(r_{\text {pupil }}\right)=\Delta \phi \cdot P_{\text {mask }} / 2 \text {. }
$$


a.)

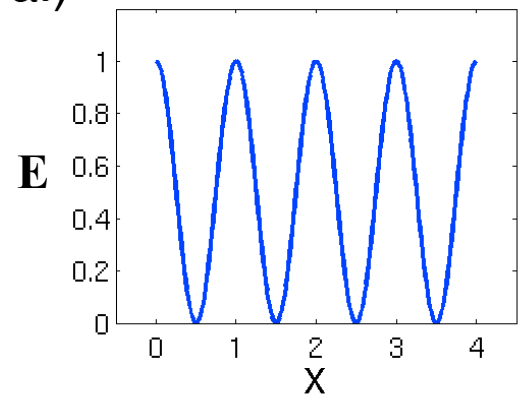

b.)

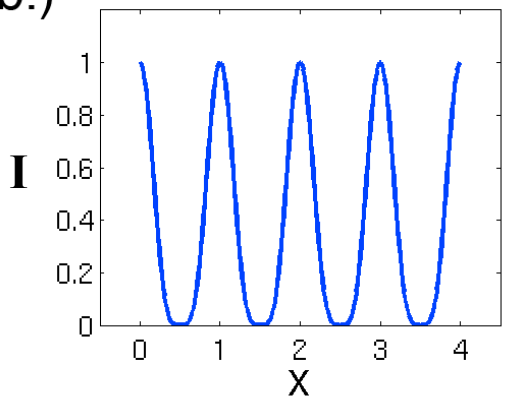

C.)

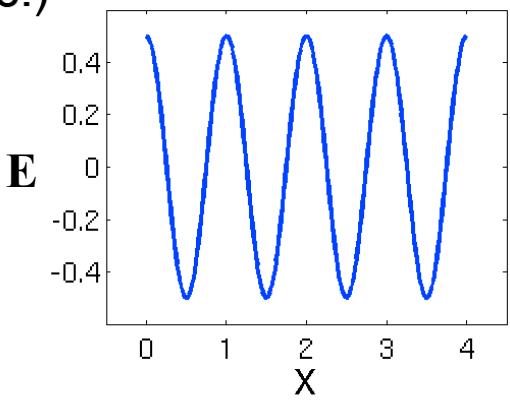

d.)

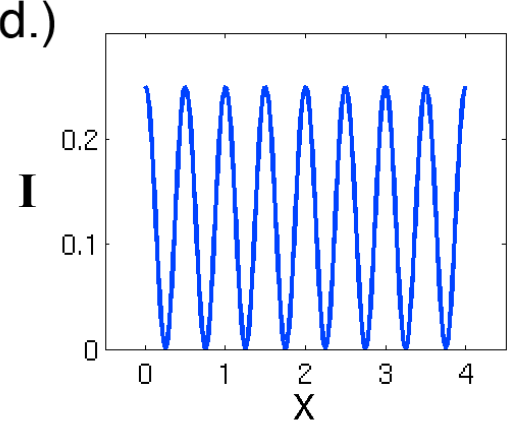

Figure 2. Illustration of frequency doubling with central obscuration (the $x$-axis is in units of mask period). a.) Aerial image electric field with no central obscuration, b.) aerial image intensity with no central obscuration, c.) aerial image electric field with central obscuration blocking zero order, d.) aerial image intensity with central obscuration blocking zero order.

The MET wavefront has previously been measured using a variety of interferometry techniques. ${ }^{5}$ The results from at-wavelength lateral shearing interferometry were used to determine the expected pattern shift in the $x$-direction as a function of normalized pupil radius based on aerial image simulations. The results are summarized in Table 1. The expected pattern shifts range from less than $1 \mathrm{~nm}$ to nearly $7 \mathrm{~nm}$, and the direction of the lateral shift changes as a function of pupil radius. These pattern shift values (along with the corresponding values for other pattern orientations) may be thought of as a "signature" for a particular wavefront. Changes in the wavefront over time as a result of aberration drift will result in a change in this signature. Therefore, this technique may serve as a method for monitoring aberration drift. A significant change in the pattern shift signature could be a signal of a potentially problematic change in the system wavefront. More detailed characterization and corrective measures could then be performed as needed.

In order to measure the amount of lateral shift in the line/space patterns in photoresist, a reference that remains stationary in the presence of aberrations is required. Ideally, this would be something like a large $(e . g ., 500 \mathrm{~nm})$ square

Table 1. Expected pattern shifts in the $x$-direction for the base MET wavefront.

\begin{tabular}{|c|c|c|}
\hline $\boldsymbol{r}_{\text {pupil }}$ & $\boldsymbol{P}_{\text {mask }}$ & Pattern Shift in Aerial Image \\
\hline 0.35 & $129 \mathrm{~nm}$ & $-6.8 \mathrm{~nm}$ \\
\hline 0.45 & $100 \mathrm{~nm}$ & $-5.3 \mathrm{~nm}$ \\
\hline 0.55 & $82 \mathrm{~nm}$ & $-4.5 \mathrm{~nm}$ \\
\hline 0.65 & $69 \mathrm{~nm}$ & $-3.2 \mathrm{~nm}$ \\
\hline 0.75 & $60 \mathrm{~nm}$ & $-2.0 \mathrm{~nm}$ \\
\hline 0.85 & $53 \mathrm{~nm}$ & $0.8 \mathrm{~nm}$ \\
\hline 0.95 & $47.4 \mathrm{~nm}$ & $3.2 \mathrm{~nm}$ \\
\hline
\end{tabular}


pattern. However, because such large features are primarily composed of relatively low spatial frequencies, the corresponding diffracted orders from the highly coherent illumination are blocked by the central obscuration and only the highest spatial frequencies (related to the edges and corners of the square) reach the wafer to form the image. This image tends to be very weak, as only a small fraction of the total diffracted energy is contained in these higher orders, and only the outline of the square is present. On the other hand, small features (which have stronger high-frequency content) tend to be more sensitive to aberrations.

As a compromise between these two extremes, the initial design uses an array of five $100 \mathrm{~nm}$ squares as a reference with which to measure lateral pattern shifts in the line/space patterns. However, this design is not perfect. The aerial image of a $100 \mathrm{~nm}$ square for an unaberrated annular pupil with a NA of 0.3 and a central obscuration with a NA of 0.1 (the same parameters as the MET optic) is shown in Figure 3. Note that the central obscuration blocks the diffracted orders corresponding to the low spatial frequencies of the square, and only the corners of the square show up in the aerial image. In addition, the aerial image of a $100 \mathrm{~nm}$ square using the MET wavefront as measured with lateral shearing interferometry is shown in Figure 4. Note that the aberrations present in the MET cause significant aerial image distortion when compared with the unaberrated case in Figure 3. This will make it difficult to establish the correct center of the reference pattern. Alternative designs are being considered.

\section{TEST MASK DESIGN}

A multilayer-coated reflective mask suitable for use in the MET tool has been designed and fabricated for testing this technique. This mask consists of many replications of a test cell. The test cell is an array of line/space patterns with different pitches designed to have a first diffraction orders at evenly spaced intervals within the pupil (normalized pupil radius values of 0.35 to 0.95 in steps of 0.1 ). Small arrays of $100 \mathrm{~nm}$ squares are interspersed with the line/space patterns to serve as pattern shift reference marks. This cell is shown in Figure 5.

The test cell in Figure 5 is present on the mask in four different rotations: $0^{\circ}, 45^{\circ}, 90^{\circ}$, and $135^{\circ}$. This will allow for "sampling" of the pupil wavefront in four different directions. These four rotated cells together form a test module, which is shown in Figure 6. This module is then repeated throughout the $600 \mu \mathrm{m} \times 200 \mu \mathrm{m}$ (in wafer dimensions) field as shown in Figure 7.

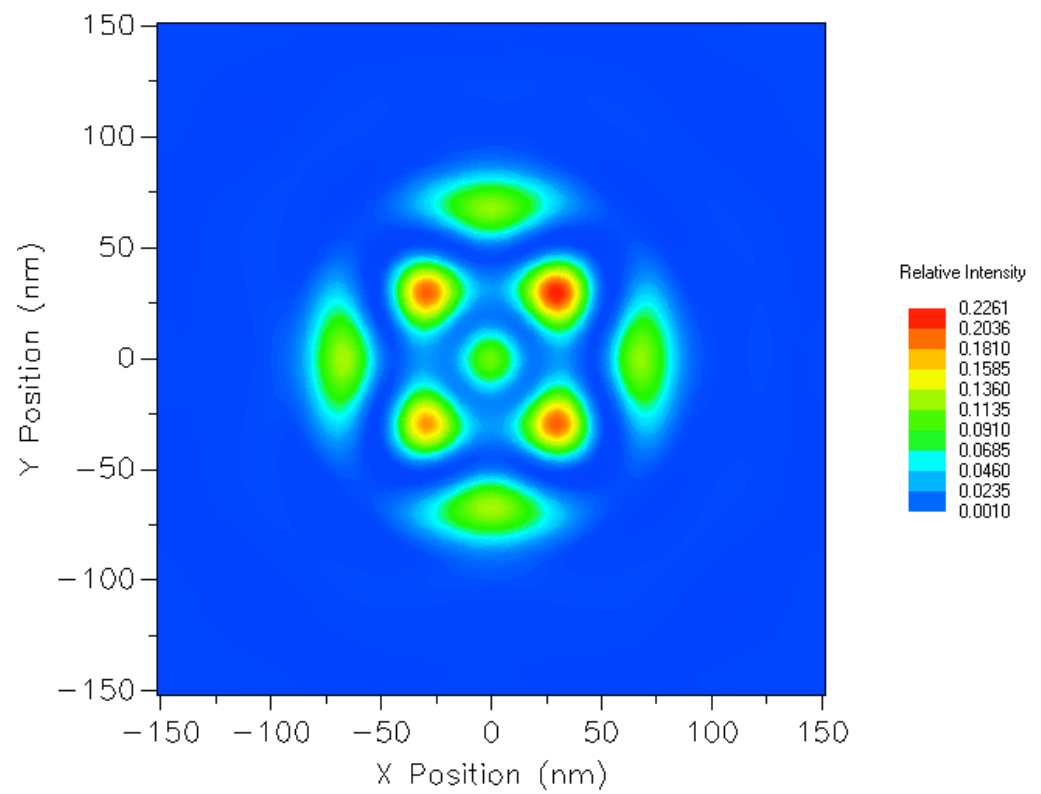

Figure 3. Simulated aerial image of a $100 \mathrm{~nm}$ square for an unaberrated annular pupil with a NA of 0.3 and a central obscuration with a NA of 0.1 (the same parameters as the MET optic). Conventional illumination with $\sigma=0.05$ was used. The diffracted orders corresponding to low spatial frequencies are blocked by the central obscuration, leaving only the highest spatial frequencies to form the image. 


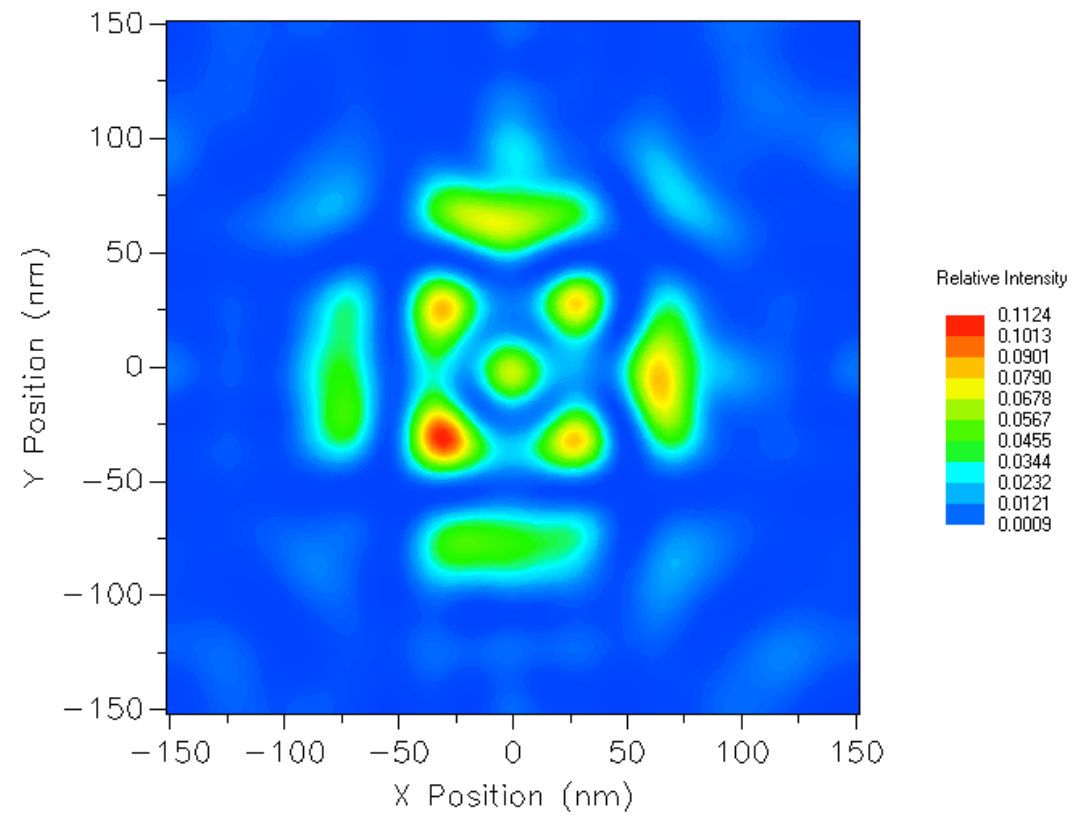

Figure 4. Simulated aerial image of a $100 \mathrm{~nm}$ square using the MET wavefront as measured using lateral shearing interferometry. Conventional illumination with $\sigma=0.05$ was used. Again, the diffracted orders corresponding to low spatial frequencies are blocked by the central obscuration, leaving only the highest spatial frequencies to form the image. The aberrations present in the MET optic are responsible for the image deformations in comparison to the unaberrated case shown in Figure 3.

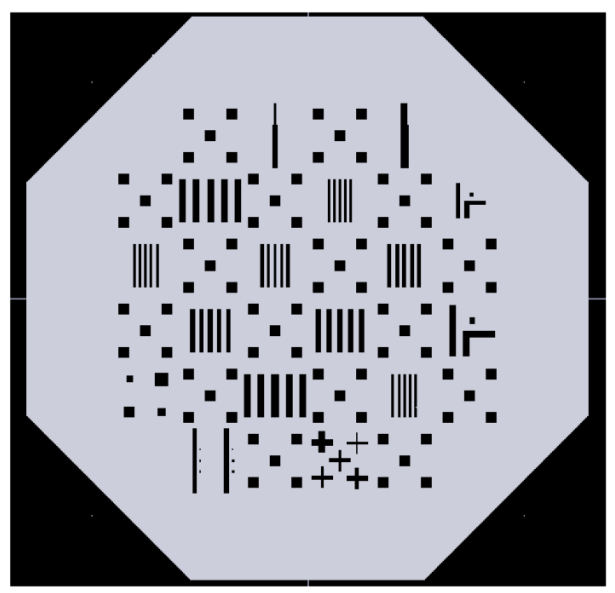

Figure 5. Test cell consisting of an array of line/space patterns with different pitches designed to have a first diffraction orders at evenly spaced intervals within the pupil (normalized pupil radius values of 0.35 to 0.95 in steps of 0.1 ). Small arrays of $100 \mathrm{~nm}$ squares are interspersed with the line/space patterns to serve as pattern shift reference marks. 

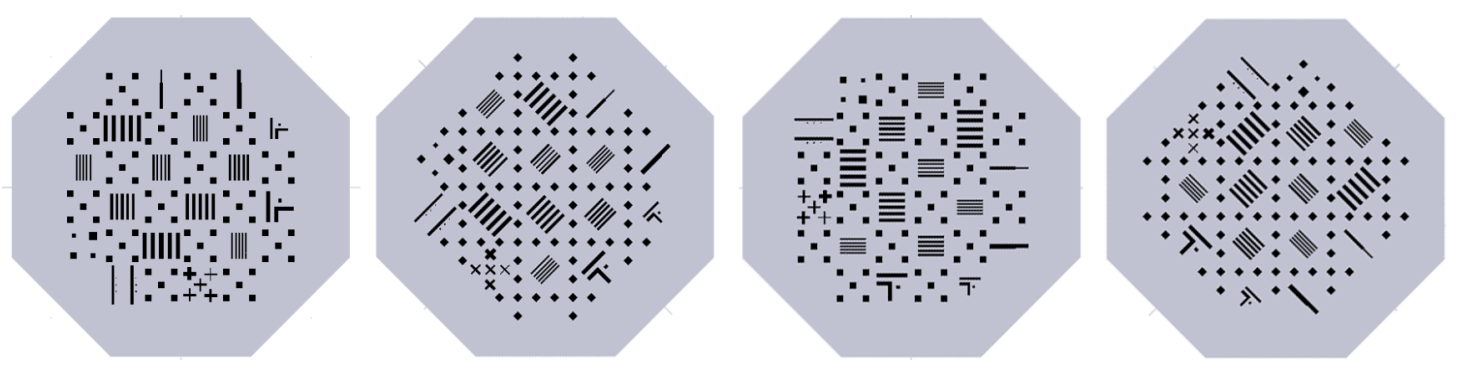

Figure 6. Aberration test module. Target orientations are (left to right) $0^{\circ}, 45^{\circ}, 90^{\circ}$, and $135^{\circ}$.

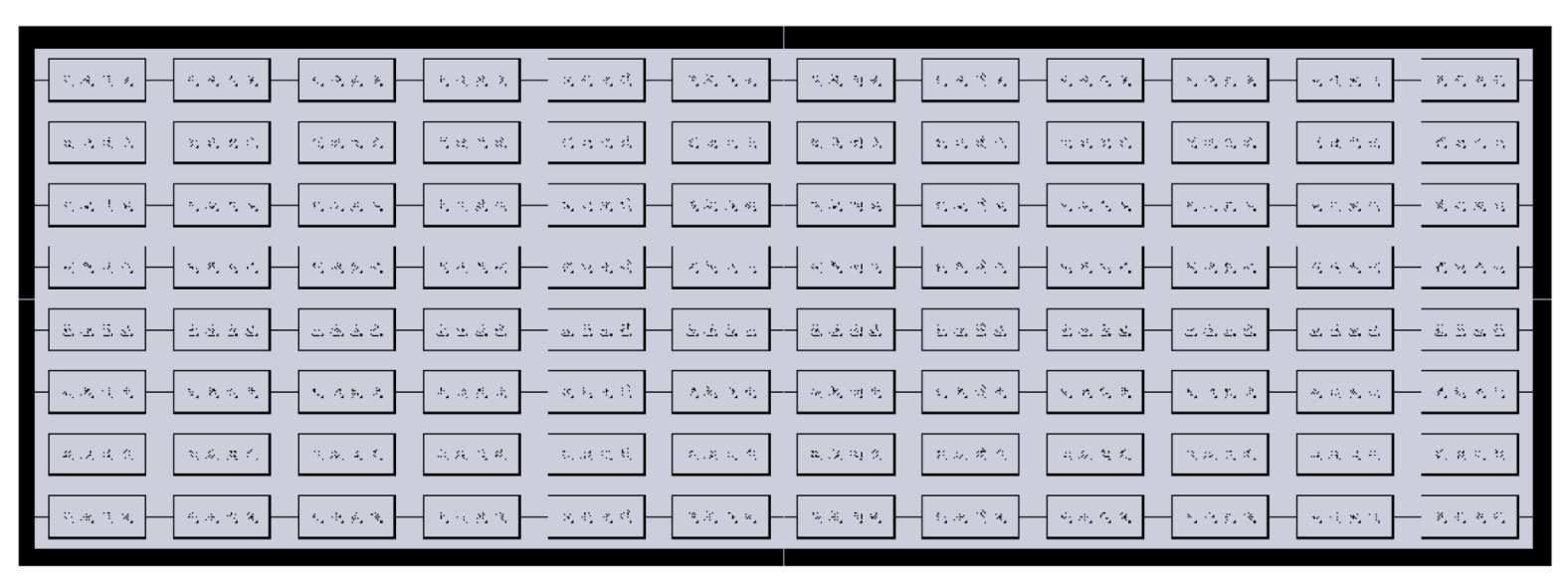

Figure 7. Test mask layout. Field size is $600 \mu \mathrm{m} \times 200 \mu \mathrm{m}$ at the wafer.

\section{PRACTICAL IMPLEMENTATION CONSIDERATIONS}

There are several practical concerns in the implementation of this aberration monitoring technique. One is the issue of exposure dose imbalance between the reference marks and the line/space patterns. Because most of the energy from the square reference patterns is in lower diffraction orders that are blocked by the central obscuration, the effective dose at the wafer for the square patterns is much lower than the dose for the line and space patterns. One possible solution to this problem is to target the exposure dose in order to print the square corners, which will result in overexposure of the line and space patterns (causing them to print as a cleared block rather than distinct lines and spaces). Additionally, the lines and spaces will be very small at the wafer (as small as $\sim 12 \mathrm{~nm}$ ) because of the frequency doubling effect described in Section 2. At the present time there are no EUV resists which can support this kind of resolution. However, as only the lateral position of the line and space patterns is important, it is not necessary to resolve individual lines. The position of the overexposed block may still serve to measure the amount of pattern shift. Therefore, the overexposure is not expected to pose a problem.

A second challenge is the development of image processing software to perform the actual measurements. Because the relative position measurements required by this technique are not supported in commercial SEM software, this would most likely be implemented as an offline software package. The software would need the capability to read a SEM image, locate both the reference structures and line and space patterns, find the center of each, and measure the relative position shifts.

One additional practical consideration is the alignment of the illumination within the pupil. For measuring odd aberrations, it is desirable to have a small illumination size at the center of the pupil. However, due to the central obscuration, the illumination pupil fill monitor in the MET cannot see this illumination pattern. Therefore, it is difficult 
to ensure that the illumination is properly centered. This is critical because any position deviation from the pupil center, in combination with radially symmetric errors including defocus, will result in lateral shifts in the line and space patterns that may be erroneously interpreted as the result of aberrations. In addition, the measurement of even aberrations requires a small illumination size at a specific off-axis location in the pupil. Again, the position of the line and space patterns at the wafer is highly sensitive to the placement of the illumination within the pupil. Achieving the required precision in illumination placement is therefore critical.

Finally, for other commercial EUV lithography systems that use a similar optic but different illuminators ${ }^{6}$, achieving the required level of partial coherence may be problematic. In the MET system at LBNL, the use of synchrotron radiation as a source provides a high degree of coherence. However, this may be more difficult to achieve in systems that use Xe gas discharge plasmas, for example.

\section{CONCLUSIONS}

A technique for monitoring drift in aberrations levels over time for centrally obscured optical systems was described. This method relies on highly coherent illumination $(\sigma \cong 0.05)$ and takes advantage of the central obscuration to block the zero diffraction order from line/space patterns, creating a two-wave interference pattern at the wafer. This pattern will shift laterally in the presence of wavefront aberrations, and the amount of shift is proportional to the amount of optical path difference between the two waves (the +1 and -1 diffracted orders) that interfere to form the image at the wafer. The blocking of the zero order leads to a frequency doubling effect in the aerial image at the wafer. Varying the pitch of the line/space patterns allows for "sampling" of the optical path difference for different locations within the pupil.

Aerial image simulations using the interferometrically-measured wavefront for an EUV optic with an annular pupil (the MET optic) were used to predict the amount of lateral shift expected for this wavefront as a function of normalized pupil radius. The shifts were on the order of 1-7 nm.

The design of reference structures intended to remain stationary within the aerial image in the presence of aberrations was also addressed. The central obscuration makes selection of an optimal reference structure difficult. A sub-optimal compromise based upon an array of five $100 \mathrm{~nm}$ squares was adopted for the initial design. Aerial image simulations were also used to predict the performance of these reference squares for both the unaberrated case and for the measured wavefront from the MET optic.

An initial test mask to implement this technique has been designed and fabricated. The mask consists of multiple test modules placed throughout the field. Each module contains four different rotations of a test structure cell consisting of the various line/space patterns and reference structures.

Finally, several challenges in implementation remain. As mentioned previously, the reference structure design is most likely sub-optimal. Also, the image processing software required to make the data analysis efficient enough for routine operation needs to be developed. The alignment of the illumination spot at the center of the pupil is difficult because the central obscuration prevents the small illumination spot from being seen by the pupil fill monitor in the MET system. Finally, other tools with a similar optical design but different illuminators may have difficulty achieving the high degree of coherence required. Each of these challenges must be addressed in order for this technique to be viable. However, if they can be met successfully, this method may provide a relatively quick way of monitoring aberration drift over time.

\section{ACKNOWLEDGMENTS}

Lawrence Berkeley National Laboratory is operated under the auspices of the Director, Office of Science, Office of Basic Energy Science, of the US Department of Energy. This work was funded by Advanced Micro Devices, Applied Materials, Atmel, Cadence, Canon, Cymer, DuPont, Ebara, Intel, KLA-Tencor, Mentor Graphics, Nikon Research, Novellus Systems, Panoramic Technologies, Photronics, Synopsis, Tokyo Electron, and the UC Discovery Grant. 


\section{REFERENCES}

1. P. Naulleau, K. A. Goldberg, E. Anderson, K. Bradley, R. Delano, P. Denham, B. Gunion, B. Harteneck, B. Hoef, H. Huang, K. Jackson, G. Jones, D. Kemp, J. A. Liddle, R. Oort, A. Rawlins, S. Rekawa, F. Salmassi, R. Tackaberry, C. Chung, L. Hale, D. Phillion, G. Sommargren, J. Taylor, "Status of EUV microexposure capabilities at the ALS using the 0.3-NA MET optic," in Emerging Lithographic Technologies VIII, R. Scott Mackay, ed., Proc. SPIE 5374, pp. 881-891, 2004.

2. P. Naulleau, K. A. Goldberg, E. Anderson, J. P. Cain, P. Denham, K. Jackson, A.-S. Morlens, S. Rekawa, F. Salmassi, "Extreme ultraviolet microexposures at the Advanced Light Source using the 0.3 numerical aperture micro-exposure tool optic," J. Vac. Sci. Tech. B, 22(6), pp. 2962-2965, Nov./Dec. 2004.

3. P. P. Naulleau, K. A. Goldberg, E. H. Anderson, J. P. Cain, P. Denham, B. Hoef, K. Jackson, A. Morlens, S. Rekawa, "EUV microexposures at the ALS using the 0.3-NA MET projection optics," in Emerging Lithographic Technologies IX, R. Scott Mackay, ed., Proc. SPIE 5751, 2005.

4. P. P. Naulleau, K. A. Goldberg, P. Batson, J. Bokor, P. Denham, and S. Rekawa, "Fourier-synthesis customcoherence illuminator for extreme ultraviolet microfield lithography," Applied Optics 42, pp. 820-826, February 2003.

5. K. A. Goldberg, P. Naulleau, P. Denham, S. B. Rekawa, K. Jackson, J. A. Liddle, E. H. Anderson, "EUV interferometric testing and alignment of the 0.3 NA MET optic," in Emerging Lithographic Technologies VIII, R. Scott Mackay, ed., Proc. SPIE 5374, pp. 64-73, 2004.

6. A. Brunton, J. Cahsmore, P. Elbourn, G. Elliner, M. Gower, P. Grünewald, M. Harman, S. Hough, N. McEntee, S. Mundair, D. Rees, P. Richards, V. Tru_ert, I. Wallhead, and M. Whitfield, "High-resolution EUV microstepper tool for resist testing and technology evaluation," in Emerging Lithographic Technologies VIII, R. Scott Mackay, ed., Proc. SPIE 5374, pp. 869-880, 2004. 